\title{
Magneto-Dielectrics in Electromagnetics: Concept and Applications
}

\author{
Hossein Mosallaei, Student Member, IEEE, and Kamal Sarabandi, Fellow, IEEE
}

\begin{abstract}
In this paper, the unique features of periodic magneto-dielectric meta-materials in electromagnetics are addressed. These materials, which are arranged in periodic configurations, are applied for the design of novel EM structures with applications in the VHF-UHF bands. The utility of theses materials are demonstrated by considering two challenging problems, namely, design of miniaturized electromagnetic band-gap (EBG) structures and antennas in the VHF-UHF bands. A woodpile EBG made up of magneto-dielectric material is proposed. It is shown that the magneto-dielectric woodpile not only exhibits band-gap rejection values much higher than the ordinary dielectric woodpile, but also for the same physical dimensions it shows a rejection band at a much lower frequency. The higher rejection is a result of higher effective impedance contrasts between consecutive layers of the magneto-dielectric woodpile structure. Composite magneto-dielectrics are also shown to provide certain advantages when used as substrates for planar antennas. These substrates are used to miniaturize antennas while maintaining a relatively high bandwidth and efficiency. An artificial anisotropic meta-substrate having $\mu_{r}>\varepsilon_{r}$, made up of layered magneto-dielectric and dielectric materials is designed to maximize the bandwidth of a miniaturized patch antenna. Analytical and numerical approaches, based on the anisotropic effective medium theory (AEMT) and the finite-difference time-domain (FDTD) technique, are applied to carry out the analyzes and fully characterize the performance of finite and infinite periodic magneto-dielectric meta-materials integrated into the EBG and antenna designs.
\end{abstract}

Index Terms-Anisotropic effective medium theory (AEMT), antenna miniaturization, electromagnetic band-gap (EBG) materials, finite-difference time-domain (FDTD) technique, magneto-dielectrics, meta-materials, periodic structures.

\section{INTRODUCTION}

$\mathbf{T}$ HE USE OF mobile wireless communication for a wide number of applications is on the rise. Depending on the application (data rate, range, etc.), the frequency, bandwidth, transmitted power, and modulation scheme of todays wireless systems may vary widely. However, independent of the application, three antenna features are always sought in a wireless system. These include: 1) compactness; 2) power efficiency; and 3) affordability.

In recent years, considerable efforts have been devoted toward miniaturization of RF electronics and development of power efficient amplifiers. However, the current state of the art in power efficient and miniaturized antennas, integrated filters, and multiplexers leaves much to be desired. Most RF passive

Manuscript received July 11, 2003; revised August 18, 2003.

The authors are with the Department of Electrical Engineering and Computer Science, University of Michigan, Ann Arbor, Ann Arbor, MI 48109-2122 USA (e-mail: hosseinm@engin.umich.edu).

Digital Object Identifier 10.1109/TAP.2004.829413 components such as filters, lumped elements, and substrates are made using high quality materials. Currently, high dielectric, low loss materials are widely used in the fabrication of miniaturized filters, diplexers, and antennas [1]-[4]. At VHF through the lower UHF band where miniaturization of RF components and antennas are highly desirable, the application of magneto-dielectric composite meta-materials is proposed. Engineering effective medium properties of a composite material by proper arrangement of constituent dielectric and magneto-dielectric materials provides more degrees of freedom in achieving desired functionalities.

Most ferrite materials are highly lossy in the VHF range and up. To achieve a low-loss ferrite with relatively high permeability $\left(\mu_{r}>10\right)$ capable of operating up to $500 \mathrm{MHz}$, researchers at Trans-Tech Inc. ${ }^{1}$ align Z-type hexaferrite ceramic material that can be found in any desired shape. Basically barium hexaferrite $\left(\mathrm{BaFe}_{12} \mathrm{O}_{19}\right)$ is blended with cobalt oxide and barium carbonate into a slurry which can be shaped using a sacrificial mold and heat treatment.

In this paper, two challenging problems in RF engineering, namely, design of EBG structures and miniaturized antennas are addressed. Dielectric band-gap materials are a class of periodic structures that can be used in the construction of high $Q$ filters and isolators among circuit subsystems [1], [2], [5], [6]. Compactness and improved band-gap rejection levels are two desired features of band-gap materials. Utilizing the magneto-dielectric material it is shown that not only can the size be reduced, but also a much higher rejection level can be obtained when compared to the dielectric-only EBG structures.

Another interesting application of magneto-dielectrics is in the area of antenna miniaturization. Antenna miniaturization using high permittivity materials as substrates has been attempted in the past [4], [7]. Although miniaturization can be achieved using high dielectric materials, there are two drawbacks. One problem stems from the fact that the field remains highly concentrated around the high permittivity region (field confinement), which results in low antenna efficiency and narrowband characteristics. The second drawback pertains to the fact that the characteristic impedance in a high permittivity medium is rather low which creates difficulties in impedance matching of the antenna. These aforementioned problems can be effectively circumvented if one uses a magneto-dielectric material. Magneto-dielectric materials can also miniaturize the antenna by the same factor however using moderate values of permittivity and permeability $\left(n=\sqrt{\mu_{r} \varepsilon_{r}}\right)$. Thus, the issue of strong field confinement is minimized and the medium is

\footnotetext{
${ }^{1}$ Trans-Tech, Inc., Adamstown, MD 21710 USA is a subsidiary of Skyworks Solutions, Inc., Woburn, MA 01801.
} 


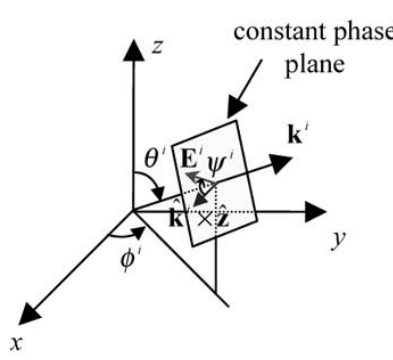

(a)

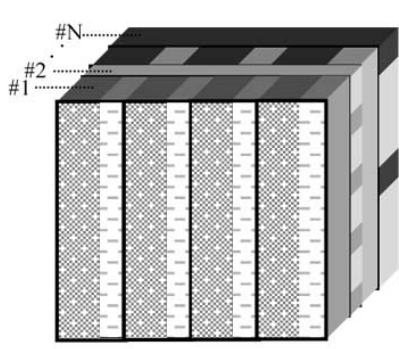

(b)

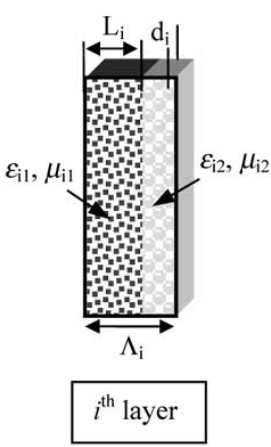

(c)

Fig. 1. 1-D periodic magneto-dielectric: (a) Plane wave excitation and its corresponding coordinate system, (b) $N$-layer periodic structure in $y$ or $z$ directions, (c) unit cell of $i$ th layer; it is assumed that this layer is periodic in $y$ and infinite in $z$ directions.

far less capacitive when compared to the dielectric-only high permittivity material. Furthermore, since the characteristic impedance of magneto-dielectric medium $\left(\eta=\eta_{0} \sqrt{\mu_{r} / \varepsilon_{r}}\right)$ is close to that of the surrounding medium $\left(\eta_{0}\right)$ it allows for ease of impedance matching over a much wider bandwidth.

To obtain the interactions of electromagnetic waves with complex finite and infinite periodic magneto-dielectric meta-materials, analytical and numerical approaches based on anisotropic effective medium theory (AEMT) and the finite-difference time-domain (FDTD) technique are used. In the AEMT method the periodic magneto-dielectric material is represented approximately as an anisotropic effective medium with permittivity and permeability tensors $\bar{\varepsilon}$ and $\bar{\mu}$, and the wave propagation characteristics are determined analytically. The FDTD full wave analysis used here allows for arbitrary placement of the FDTD boundary using a periodic boundary condition (PBC) or a perfectly matched layer (PML) or a combination of these depending on the problem at hand.

\section{PROBlem's Formulation}

In this section, the formulations necessary for characterizing composite magneto-dielectric meta-materials are developed. Depending on the complexity of the structure under study, analytical or numerical methods will be used. The analytical model has the advantage of providing insight into the nature of EM waves interacting with the medium, whereas, the numerical method will be used for complicated structures for which an analytical solution does not exist.

\section{A. AEMT}

In construction of magneto-dielectric structures one may consider one-dimensional (1-D) periodic layers made up of dielectric and magnetic materials. The layers can be stacked on top of each other with an arbitrary orientation direction. One can use a quasistatic mixing model [8], [9] to obtain the effective medium properties of periodic magneto-dielectrics. However, this approach is only applicable when the period is much smaller than a wavelength (quasistatic region). In what follows, a general formulation for periodic magneto-dielectric media is presented that provides accurate results for period values as high as $\lambda / 2$.

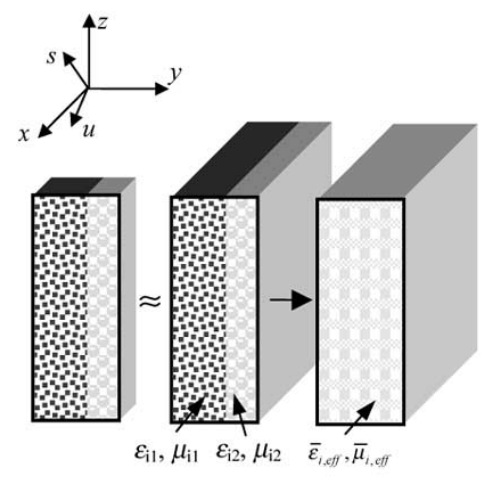

(a)

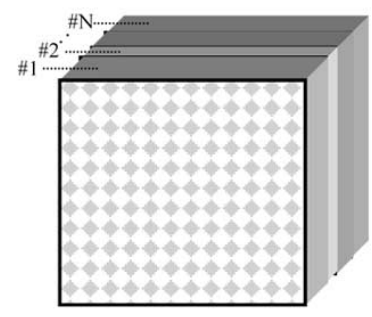

(b)
Fig. 2. Anisotropic effective material model for the 1-D periodic magneto-dielectric: (a) A unit cell is viewed as a slice of slab that is infinite in $x$ and $z$, and the fact that a medium of periodic slabs can be modeled by an effective magneto-dielectric material and (b) $N$-layer anisotropic effective $\bar{\varepsilon}-\bar{\mu}$ media representing the original layered $1-\mathrm{D}$ periodic magneto-dielectric [Fig. 1(b)].

AEMT is a very capable method for analyzing periodic structures with relatively large periods. It is shown that 1-D periodic dielectric layers can be viewed as a uniaxial anisotropic medium with the optical axis along the periodic direction. The relationship between the effective constitutive parameters and the geometrical and electrical parameters of the periodic medium composed of only dielectric materials was described in [10]. To obtain the performance of a stack of magneto-dielectric periodic layers, AEMT must be extended to include magnetic materials as well.

The geometry of an $N$-layer 1-D periodic structure stacked along the $x$ direction and illuminated by an arbitrary incident plane wave is depicted in Fig. 1. Each periodic layer is assumed to be made of both dielectric and magnetic materials with periodicity $\Lambda_{i}$ (layers are periodic in the $y$ or $z$ directions), where the subscript $i$ represents the $i$ th region $(i=1,2, \ldots, N)$. The direction and polarization of the incident wave and its corresponding coordinate system are defined in Fig. 1(a). Note that a linear polarization is denoted by the angle $\psi^{i}$ between the electric field and a reference direction defined by $\mathbf{k}^{i} \times \hat{\mathbf{z}}$, where $\mathbf{k}^{i}$ is the incident wave propagation direction.

To obtain the effective constitutive parameters for each layer the medium is assumed to be infinite in the $x$ direction as illustrated in Fig. 2(a). This assumption is applied only where 
the effective parameters of the layers are obtained and in the remainder of the analysis the actual thickness of layers is considered. This appears to be a very good approximation even when the layers are relatively thin. The 1-D periodic medium is then analyzed by considering the possible solution of Maxwell's equations in the absence of any source. This is accomplished by invoking the Floquet periodic boundary conditions in addition to the traditional electric and magnetic field boundary conditions [11], [12]. Examining the possible modes of propagation it is shown that the medium acts as a uniaxial anisotropic material. The original structure [Fig. 1(b)] can then be reduced to an $N$-layer medium composed of anisotropic layers [Fig. 2(b)] with effective constitutive parameters given by the terms $\bar{\varepsilon}_{i \text {,eff }}$ and $\bar{\mu}_{i, \text { eff }}$, whose response to any arbitrary source can be analytically determined.

To demonstrate the procedure outlined above, consider an arbitrary incident plane wave illuminating a periodic layer with periodicity in the $y$ direction and infinite in the $x$ and $z$ directions. The direction of propagation of the incident wave is given by

$$
\mathbf{k}^{i}=\hat{\mathbf{x}} k_{x}^{i}+\hat{\mathbf{y}} k_{y}^{i}+\hat{\mathbf{z}} k_{z}^{i}=\hat{\mathbf{y}} k_{y}^{i}+\hat{\mathbf{s}} k_{s}^{i}
$$

where $\hat{\mathbf{s}} k_{s}^{i}$ is a transverse vector in $x-z$ plane. Since the structure is symmetric in the $x-z$ plane, the electromagnetic fields can be separated into $E_{u}$ and $H_{u}$ polarizations, where $\hat{\mathbf{u}}=\hat{\mathbf{y}} \times \hat{\mathbf{s}}$. The $\mathrm{EM}$ waves for the electric and magnetic polarizations in one unit cell of the $i$ th layer must satisfy the scalar wave equation

$$
\begin{array}{ll}
\left(\frac{\partial^{2}}{\partial y^{2}}+\frac{\partial^{2}}{\partial s^{2}}+\mu_{i 1} \varepsilon_{i 1} k_{0}^{2}\right) \Psi_{i 1}(y, s)=0, & 0 \leq y \leq L_{i} \\
\left(\frac{\partial^{2}}{\partial y^{2}}+\frac{\partial^{2}}{\partial s^{2}}+\mu_{i 2} \varepsilon_{i 2} k_{0}^{2}\right) \Psi_{i 2}(y, s)=0, & L_{i} \leq y \leq \Lambda_{i}
\end{array}
$$

where $\Psi(y, s)$, depending on the polarization, can be $E_{u}(y, s)$ or $H_{u}(y, s)$. The solution to (2) is found to be

$$
\begin{aligned}
& \Psi_{i 1}(y, s)=\left(A e^{-j k_{i 1, y} y}+B e^{j k_{i 1, y} y}\right) e^{-j k_{i s} s} \\
& \Psi_{i 2}(y, s)=\left(C e^{-j k_{i 2, y} y}+D e^{j k_{i 2, y} y}\right) e^{-j k_{i s} s}
\end{aligned}
$$

where

$$
\begin{aligned}
& k_{i 1, y}^{2}+k_{i s}^{2}=\mu_{i 1} \varepsilon_{i 1} k_{0}^{2} \\
& k_{i 2, y}^{2}+k_{i s}^{2}=\mu_{i 2} \varepsilon_{i 2} k_{0}^{2} .
\end{aligned}
$$

The continuity and periodicity (Floquet theorem) boundary conditions are next applied for determining the natural modes in the periodic structure for both polarizations. For $E_{u}$ polarization the application of these boundary conditions results in the following transcendental equation:

$$
\begin{aligned}
& -\left(\frac{\mu_{i 2} k_{i 1, y}}{\mu_{i 1} k_{i 2, y}}+\frac{\mu_{i 1} k_{i 2, y}}{\mu_{i 2} k_{i 1, y}}\right) \operatorname{Sin}\left(k_{i 1, y} L_{i}\right) \operatorname{Sin}\left(k_{i 2, y}\left(\Lambda_{i}-L_{i}\right)\right) \\
& +2 \operatorname{Cos}\left(k_{i 1, y} L_{i}\right) \operatorname{Cos}\left(k_{i 2, y}\left(\Lambda_{i}-L_{i}\right)\right)=2 \operatorname{Cos}\left(k_{y}^{i} \Lambda_{i}\right) .
\end{aligned}
$$

The application of duality to (5) determines the following transcendental equation for the $H_{u}$ polarization case:

$$
\begin{aligned}
& -\left(\frac{\varepsilon_{i 2} k_{i 1, y}}{\varepsilon_{i 1} k_{i 2, y}}+\frac{\varepsilon_{i 1} k_{i 2, y}}{\varepsilon_{i 2} k_{i 1, y}}\right) \operatorname{Sin}\left(k_{i 1, y} L_{i}\right) \operatorname{Sin}\left(k_{i 2, y}\left(\Lambda_{i}-L_{i}\right)\right) \\
& +2 \operatorname{Cos}\left(k_{i 1, y} L_{i}\right) \operatorname{Cos}\left(k_{i 2, y}\left(\Lambda_{i}-L_{i}\right)\right)=2 \operatorname{Cos}\left(k_{y}^{i} \Lambda_{i}\right) .
\end{aligned}
$$

From (4a) and (4b), it can be shown that $k_{i 1, y}^{2}-k_{i 2, y}^{2}=$ $\left(\mu_{i 1} \varepsilon_{i 1}-\mu_{i 2} \varepsilon_{i 2}\right) k_{0}^{2}$, which together with (5) or (6) provide the solutions for $k_{i 1, y}, k_{i 2, y}$, and $k_{i s}$ for the $E_{u^{-}}$or $H_{u^{-}}$ polarization cases, respectively.

The electromagnetic behavior of the $i$ th periodic medium for the dominant mode can be modeled with a uniaxial effective magneto-dielectric slab. In a uniaxial anisotropic material with the optical axis along the $y$ direction $\bar{\varepsilon}$ and $\bar{\mu}$ tensors take the following forms:

$$
\begin{aligned}
& \bar{\varepsilon}=\varepsilon_{0}\left[\begin{array}{ccc}
\varepsilon_{o} & 0 & 0 \\
0 & \varepsilon_{e} & 0 \\
0 & 0 & \varepsilon_{o}
\end{array}\right] \\
& \bar{\mu}=\mu_{0}\left[\begin{array}{ccc}
\mu_{o} & 0 & 0 \\
0 & \mu_{e} & 0 \\
0 & 0 & \mu_{o}
\end{array}\right]
\end{aligned}
$$

and the dispersion relations for $E_{u}$ or $H_{u}$ polarizations are given by

$$
\begin{aligned}
& \frac{k_{y}^{2}}{\mu_{o} \varepsilon_{o}}+\frac{k_{s}^{2}}{\mu_{e} \varepsilon_{o}}=k_{0}^{2} \\
& \frac{k_{y}^{2}}{\mu_{o} \varepsilon_{o}}+\frac{k_{s}^{2}}{\mu_{o} \varepsilon_{e}}=k_{0}^{2}
\end{aligned}
$$

where subscripts " $e$ " and " $o$ " denote the extraordinary and ordinary waves, respectively [13]. It should be pointed out that in an electric and magnetic uniaxial medium both ordinary and extraordinary waves are independent of incident wave polarizations. The $i$ th periodic layer can be thus presented as an anisotropic material if $k_{i 1, y}, k_{i 2, y}$, and $k_{i s}$ satisfy (8) for both polarizations. The parameters $\varepsilon_{i o}, \varepsilon_{i e}, \mu_{i o}$, and $\mu_{i e}$ of the $i$ th effective medium can be obtained from

$$
\begin{aligned}
& \varepsilon_{i o}=\varepsilon_{i 1}+\frac{1}{k_{0}^{2}}\left(k_{y}^{i^{2}}-k_{i 1, y}^{2}\right) \\
& \varepsilon_{i e}=\frac{\varepsilon_{o}}{\varepsilon_{o}-\left(\frac{k_{y}^{i}}{k_{0}}\right)^{2}}\left(\varepsilon_{i 1}-\left(\frac{k_{i 1, y}}{k_{0}}\right)^{2}\right) \\
& \mu_{i o}=\mu_{i 1}+\frac{1}{k_{0}^{2}}\left(k_{y}^{i^{2}}-k_{i 1, y}^{2}\right) \\
& \mu_{i e}=\frac{\mu_{o}}{\mu_{o}-\left(\frac{k_{y}^{i}}{k_{0}}\right)^{2}}\left(\mu_{i 1}-\left(\frac{k_{i 1, y}}{k_{0}}\right)^{2}\right)
\end{aligned}
$$

for a given value of $k_{y}^{i}$. Although effective $\bar{\varepsilon}$ and $\bar{\mu}$ appear to be a function of the angle of incidence, the numerical solution shows 
this dependence on incident angle is very small even when $L$ is as large as $\lambda / 2$ [10]. In the special case where $\Lambda_{i} \ll \lambda_{i}\left(\lambda_{i}\right.$ is the wavelength in $i$ th layer) the above formulation simplifies to the well-known quasistatic solution

$$
\begin{aligned}
\varepsilon_{i o} & =\varepsilon_{i 1} \frac{L_{i}}{\Lambda_{i}}+\varepsilon_{i 2}\left(1-\frac{L_{i}}{\Lambda_{i}}\right) \\
\frac{1}{\varepsilon_{i e}} & =\frac{1}{\varepsilon_{i 1}} \frac{L_{i}}{\Lambda_{i}}+\frac{1}{\varepsilon_{i 2}}\left(1-\frac{L_{i}}{\Lambda_{i}}\right) \\
\mu_{i o} & =\mu_{i 1} \frac{L_{i}}{\Lambda_{i}}+\mu_{i 2}\left(1-\frac{L_{i}}{\Lambda_{i}}\right) \\
\frac{1}{\mu_{i e}} & =\frac{1}{\mu_{i 1}} \frac{L_{i}}{\Lambda_{i}}+\frac{1}{\mu_{i 2}}\left(1-\frac{L_{i}}{\Lambda_{i}}\right) .
\end{aligned}
$$

It is also interesting to note that the expressions for the effective permittivity and permeability are separable, as expected. That is $\varepsilon_{o}$ and $\varepsilon_{e}$ are only functions of $\varepsilon_{1}$ and $\varepsilon_{2}$ and $\mu_{o}$ and $\mu_{e}$ are only functions of $\mu_{1}$ and $\mu_{2}$.

Therefore, utilizing the generalized AEMT, the layers of the 1-D periodic magneto-dielectric can be modeled with as an anisotropic media with the $\bar{\varepsilon}_{i \text {,eff }}$ and $\bar{\mu}_{i, \text { eff }}$ tensors [Fig. 2(b)] and the stack of periodic medium can be analytically characterized (see Appendix A).

\section{B. FDTD Technique}

In Section II-A the AEMT was applied to provide an analytic formulation that describes the behavior of 1-D periodic layers. However, in order to accurately characterize the performance of electromagnetic waves in more complex periodic magneto-dielectric meta-materials a more general technique is needed. Here, a powerful method based on the FDTD approach is applied [14]. The FDTD in conjunction with the PBC and PML walls [15], [16] allows for characterizing plane wave propagation within an arbitrary 3-D periodic structure. The FDTD method is appropriate for this application as it can provide the EM behavior of the magneto-dielectrics over a wide frequency range. Prony's extrapolation scheme is also integrated to efficiently expedite the computational time of the FDTD formulation. The FDTD code used here is capable of analyzing both finite and infinite periodic magneto-dielectrics.

\section{NOVEl APPliCATIONS OF MAGNETO-Dielectrics}

In this section, the feasibility of designing periodic magneto-dielectric meta-materials useful for $\mathrm{RF}$ and wireless applications is demonstrated. Two specific examples are considered: (a) electromagnetic band-gap (EBG) structures with superior properties, and (b) substrate design for miniaturized wideband planar antennas with enhanced performance.

The structures are constructed utilizing a typical magneto-dielectric (hexaferrite) recently manufactured by Trans-Tech. A sample of high performance $Z$-type hexaferrite is measured using an Agilent E4991A impedance analyzer to obtain the material permeability. However, establishing the correct dielectric constant for the medium requires an unambiguous measurement. Dielectric measurement methods using coaxial transmission lines and the impedance analyzer with relatively

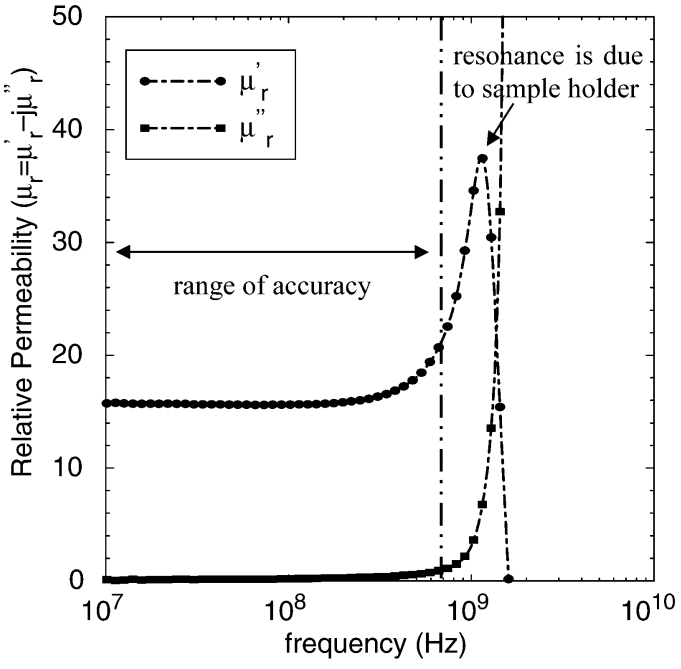

Fig. 3. Measured complex permeability of an aligned hexaferrite material manufactured by Trans-Tech Inc.

thick specimens give high numbers because of the influence of permeability and are not very reliable. The best approach is to construct a quarter wave coaxial resonator and then obtain the resonant frequency that is proportional to the square root of permeability and permittivity. From that, and substituting for the permeability, one can evaluate the permittivity. Also, by measuring the total $Q$ of the material and given the magnetic loss tangent, the dielectric loss tangent can be determined.

Fig. 3 shows the magnetic behavior $\left(\mu_{r}\right)$ of a typical magneto-dielectric material manufactured by Trans-Tech. Note that the measured results are valid up to $1 \mathrm{GHz}$ and the observed peak around this frequency is basically not due to a material resonance but rather to the device deficiency and measurement equipment. The minimum material resonance of hexaferrite should occur no lower than about $3 \mathrm{GHz}$. In the VHF range (up to $300 \mathrm{MHz}$ ) the relative permeability is found to be approximately $\mu_{r} \approx 16$ and the material has a low magnetic loss tangent of about 0.02 . The dielectric constant for this material is about $\varepsilon_{r} \approx 16$ and the dielectric loss tangent is around 0.002 . The Trans-Tech material is used in the construction of designed magneto-dielectrics presented in Sections III-A and B.

\section{A. EBG Structures}

EBG structures are abundant in nature and show interesting and useful phenomena. In particular, characteristics such as frequency stop-band, pass-band and band-gap can be identified [5], [6]. Generally speaking, EBG materials are 3-D periodic structures that prevent the propagation of electromagnetic waves in a specified band of frequency for all angles and for all polarization states.

A woodpile EBG, proposed by Ho et al. [17], is a periodic structure capable of producing complete transmission band-gap regions. In the design presented by Ho et al. only dielectric materials are used to obtain the band-gap structure. In this work, a woodpile structure composed of magneto-dielectric material is considered which shows superior band-gap characteristics.

Fig. 4 depicts the geometry of an eight-layer woodpile structure of dielectric and magneto-dielectric materials. The even- 


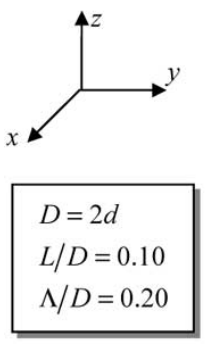

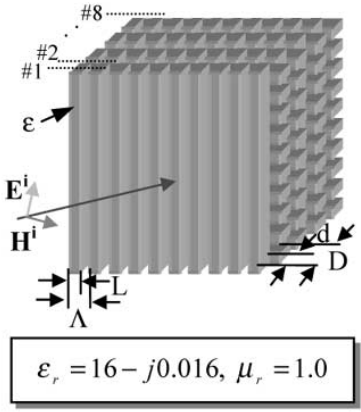

(a)

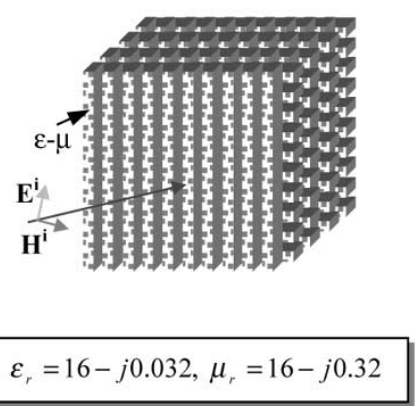

(b)

Fig. 4. Eight-layer woodpile EBG structure (periodic in $y$ - $z$ directions); even layers are rotated $90^{\circ}$ relative to the odd layers: (a) Dielectric woodpile ( $\varepsilon$ material), (b) Magneto-dielectric woodpile ( $\varepsilon-\mu$ material).

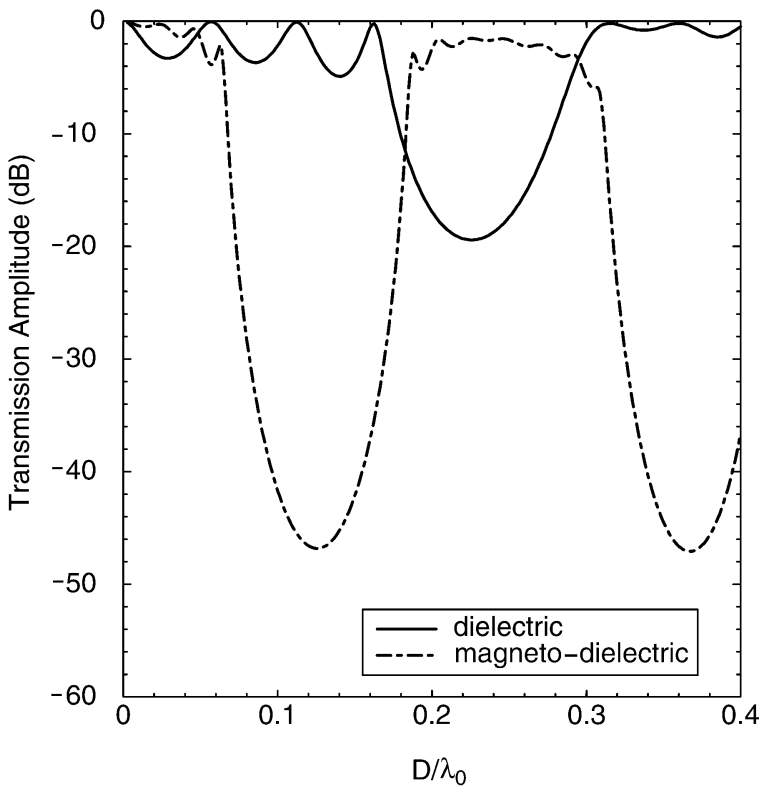

(a)

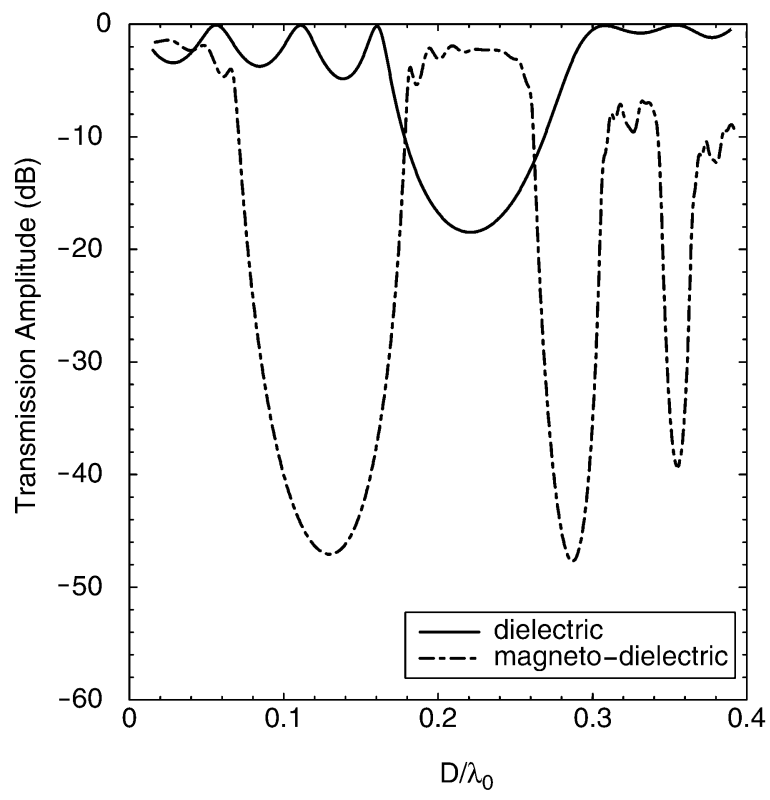

(b)

Fig. 5. Transmission coefficients of the dielectric and magneto-dielectric woodpile EBG for an arbitrary incident plane wave with $\theta^{i}=90^{\circ}, \phi^{i}=140^{\circ}$, and $\psi^{i}=60^{\circ}$ : (a) AEMT analytical results, (b) FDTD numerical results. The magneto-dielectric woodpile shows the lower band reject frequency (reduced size) and enhanced rejection level.

numbered layers are similar to the odd-numbered layers except that they are rotated by $90^{\circ}$. The AEMT analytical technique and FDTD full wave analysis are applied to characterize the structure by examining the transmission coefficient through the woodpile for an arbitrary incident plane wave with $\theta^{i}=90^{\circ}$, $\phi^{i}=140^{\circ}$ and a linear polarization specified by angle $\psi^{i}=$ $60^{\circ}$, where $\psi^{i}$ is the angle between the electric field and reference direction $\hat{\mathbf{k}}^{i} \times \hat{\mathbf{z}}$.

Fig. 5(a) and (b) show the transmission coefficients for two different woodpiles, namely, dielectric and magneto-dielectric in a free space background as a function of normalized frequency $D / \lambda_{0}(D=2 d$ is the periodicity in the $x$ direction) using the AEMT and FDTD approaches, respectively. In these simulations the filling factor $L / \Lambda$ is 0.50 and $\Lambda / D=0.20$. The dielectric woodpile is constructed from dielectric material with $\varepsilon_{r}=16$ (loss tangent $\operatorname{tg} \delta_{l}=0.001$ ) and for the magneto-dielectric $\varepsilon_{r}=\mu_{r}=16$ (Z-type hexaferrite) is used. All layers have equal thicknesses of $d$. As observed, for the dielec- tric material the AEMT and FDTD results have excellent agreement. For the magneto-dielectric since $\varepsilon_{r}=16$ and $\mu_{r}=16$ (material wavelength is $\lambda=\lambda_{0} / 16$ ), the AEMT has a good agreement with FDTD only up to the normalized frequencies of about $D / \lambda_{0}=0.22$ where $\Lambda=0.70 \lambda$. For periodicity $\Lambda>\lambda / 2$ more than one Bragg mode exists and the more general full wave analysis must be applied. The results however shows the usefulness of AEMT in analyzing periodic structures having relatively large periods, of up to $\Lambda=\lambda / 2$.

As observed in Fig. 5, the dielectric woodpile EBG generates a band-gap region at the normalized frequency $D / \lambda_{0}=$ 0.22 with in-band rejection of about $-18 \mathrm{~dB}$. In this design the rectangular grains of the odd-numbered layers $(\# 1,3,5,7)$ are parallel to the electric field and hence produce a larger effective permittivity $\left(\varepsilon_{o}\right)$ than the even-numbered layers $\left(\varepsilon_{e}\right)$. Therefore, a wave impedance contrast exists between the layers $\left(\eta_{2} / \eta_{1}=\sqrt{\varepsilon_{o} / \varepsilon_{e}}\right)$ that is responsible for the rejection level at the band-gap region. 


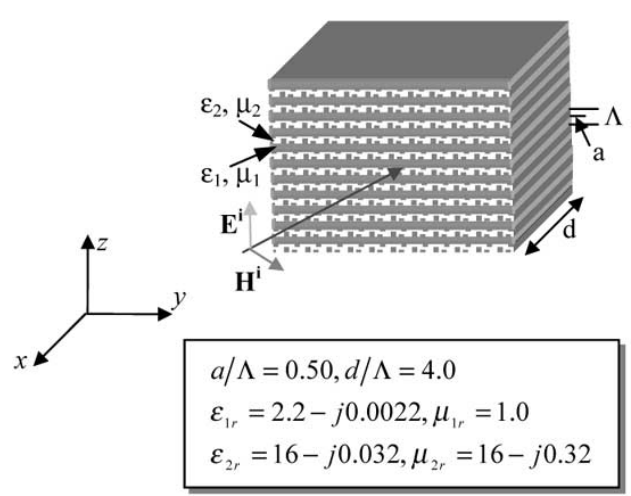

(a)

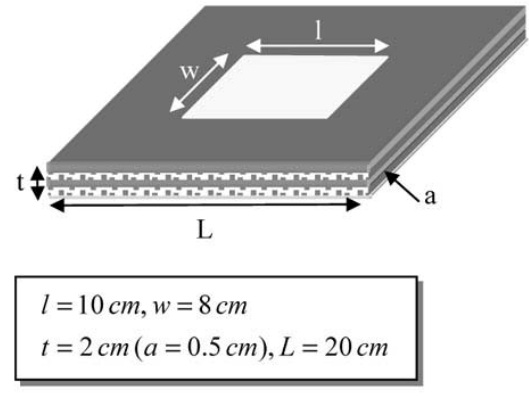

(b)

Fig. 6. (a) Composite periodic dielectric and magneto-dielectric structure (periodicity in $z$ directions). (b) A patch antenna over the engineered magneto-dielectric meta-substrate.

The magneto-dielectric band-gap shown in Fig. 4(b) is demonstrated to have much higher rejection values (see Fig. 5). In this case, the wave impedance contrast between odd- and even-numbered layers is much higher that of the dielectric woodpile. Basically when the electric field is parallel to the rectangular grains of the odd-numbered layers the electric field sees a high effective $\varepsilon\left(\varepsilon_{o}\right)$ and the magnetic field sees a low effective $\mu\left(\mu_{e}\right)$. This situation is reversed for the even-numbered layers and thus, the impedance contrast $\eta_{2} / \eta_{1}=\sqrt{\left(\mu_{o} / \varepsilon_{e}\right) \cdot\left(\varepsilon_{o} / \mu_{e}\right)}$ is much higher. The in-band rejection level of the magneto-dielectric woodpile is $-47 \mathrm{~dB}$. Also since the effective index of refraction is higher, the band-gap location is shifted down to the normalized frequency of $D / \lambda_{0}=0.13$. Hence, utilizing magneto-dielectric materials one can achieve band-gap structures that are more compact and can produce better band-gap rejection levels.

\section{B. Antenna Miniaturization}

Smaller physical size, wider bandwidth, and higher efficiency are three desired characteristics of antennas for mobile systems. Antenna miniaturization can be simply achieved by printing a patch antenna over a high permittivity substrate [4]. However, because of the strong capacitive coupling between the antenna and the antenna's ground plane its performance is considerably degraded. To overcome this problem, instead of using a high dielectric material (only $\varepsilon$ ) one can use magneto-dielectric (both $\varepsilon$ and $\mu$ ) substrates. This way by choosing moderate values of $\varepsilon_{r}$ and $\mu_{r}$ the same miniaturization factor $\left(n=\sqrt{\mu_{r} \varepsilon_{r}}\right)$ can be achieved while the strong coupling between the antenna and ground plane is reduced. In other words, the capacitive property of the resonant antenna is decreased and by addition of $\mu_{r}$ some inductance is introduced that can further counteract the capacitive behavior. This will improve both the efficiency and bandwidth of the antenna.

It has been shown by Hansen and Burke [18], that the zeroorder bandwidth for an antenna over a magneto-dielectric substrate with thickness $t$ can be approximated by

$$
B W \approx \frac{96 \sqrt{\frac{\mu_{r}}{\varepsilon_{r}}} \frac{t}{\lambda_{0}}}{\sqrt{2}\left[4+17 \sqrt{\mu_{r} \varepsilon_{r}}\right]} .
$$

Thus, for a given miniaturization factor (constant $\sqrt{\mu_{r} \varepsilon_{r}}$ ) the antenna bandwidth can be enhanced by increasing $\mu_{r} / \varepsilon_{r}\left(\mu_{r}>\right.$ $\left.\varepsilon_{r}\right)$. Of course the application of the natural $Z$-type hexaferrite with $\varepsilon_{r}=\mu_{r}=16$ can provide a very small antenna $\left(\lambda_{0} / 32\right)$ of a reasonable bandwidth (much larger than using $\varepsilon_{r}=256$ ). But if a miniaturization of less than 16 is acceptable a much better bandwidth can be achieved.

To demonstrate this, consider a 1-D periodic configuration of dielectric $\left(\varepsilon_{r}=2.2\right.$, loss tangent $\left.t g \delta_{l}=0.001\right)$ and magneto-dielectric layers (same thicknesses) as shown in Fig. 6(a). A patch antenna printed on this engineered meta-substrate [Fig. 6(b)] produces an electric field $\left(E_{z}\right)$ perpendicular to the interface of the layers and a magnetic field $\left(H_{x}\right)$ parallel to the interface. As shown before [(11b) and (12a)] the composite substrate is anisotropic with effective permittivity equal to $\varepsilon_{e}$ and effective permeability equal to $\mu_{o}$. Therefore, we can design $\mu_{\text {or }}>\varepsilon_{\text {er }}$. Using equal thickness for each layer $\varepsilon_{\mathrm{er}}=3.87$ and $\mu_{\mathrm{or}}=8.50$ is obtained. This will provide a miniaturization factor greater than 5 with extremely enhanced antenna bandwidth as will be illustrated next.

The FDTD code is applied to characterize the effective parameters of the layered periodic structure [Fig. 6(a)] and obtain the transmission amplitude and phase of an oblique incident plane wave with $\theta^{i}=90^{\circ}, \phi^{i}=150^{\circ}$, and polarization state $\psi^{i}=90^{\circ}\left(E_{z}, H_{x}, H_{y}\right)$. The results are presented in Fig. 7(a) and (b). It is determined that the performance of the periodic layered structure is almost equivalent to the behavior of a homogeneous magneto-dielectric material with $\varepsilon_{\text {er }}=3.84-j 0.004$ and $\mu_{\mathrm{or}}=8.61-j 0.16$ as shown in Fig. 7. Notice that the periodic structure has an electrically small period at the desired frequency and thus, the above effective parameters $\left(\varepsilon_{e}, \mu_{o}\right)$ are in very good agreement with those estimated from $(11 \mathrm{~b})$ and (12a).

A patch antenna with size $10 \mathrm{~cm} \times 8 \mathrm{~cm}$ is printed on the four layer dielectric and magneto-dielectric materials with thickness $2 \mathrm{~cm}$ illustrated in Fig. 6(b). The size of the ground plane is $20 \mathrm{~cm} \times 20 \mathrm{~cm}$. The FDTD is applied to characterize the finite-size patch antenna design. The return loss and radiation patterns are shown in Fig. 8. The antenna resonance is at $f_{0}=277 \mathrm{MHz}$ and it provides a wide bandwidth of about $B W=3.2 \%$. The size of the antenna is around $0.09 \lambda_{0}$ with a miniaturization factor of 5.4. The directivity 


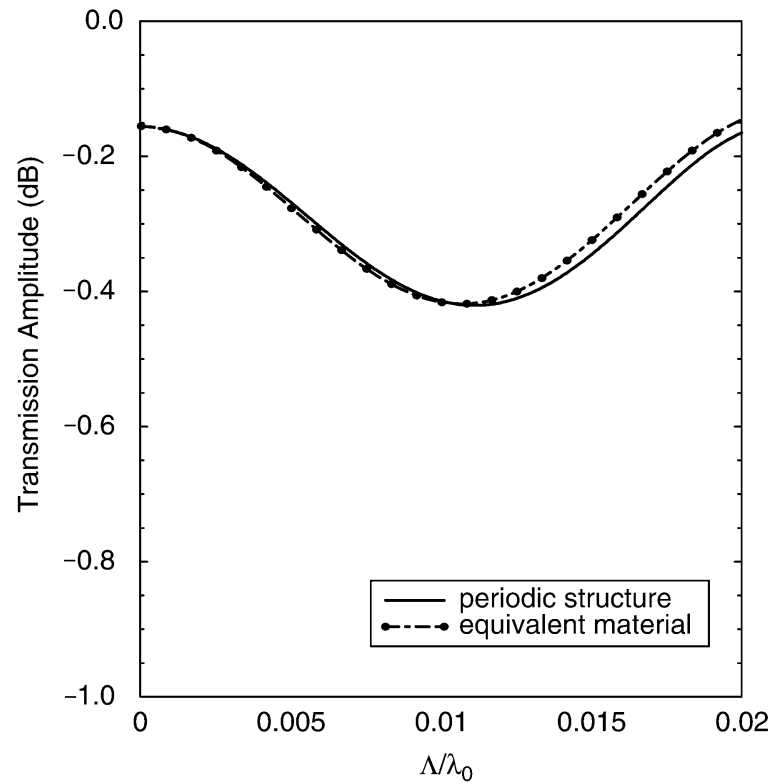

(a)

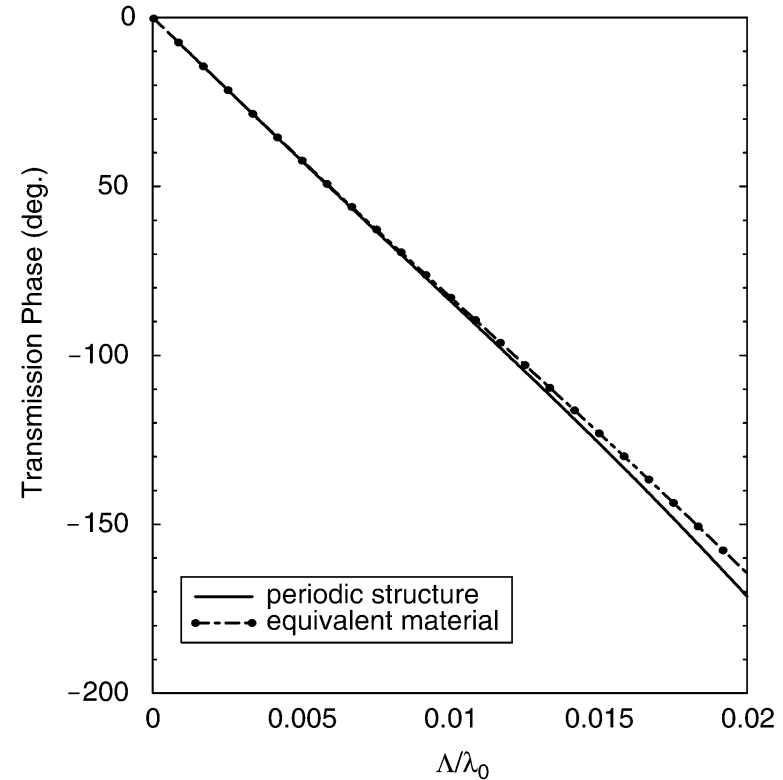

(b)

Fig. 7. Transmission coefficient of composite periodic dielectric and magneto-dielectric structure for an arbitrary incident plane wave with $\theta^{i}=90^{\circ}, \phi^{i}=150^{\circ}$, and $\psi^{i}=90^{\circ}$ : (a) Transmission amplitude and (b) transmission phase. The EM behavior of periodic structure is almost equivalent to a homogeneous material with $\varepsilon_{\mathrm{er}}=3.84-j 0.004$ and $\mu_{\mathrm{or}}=8.61-j 0.16$.

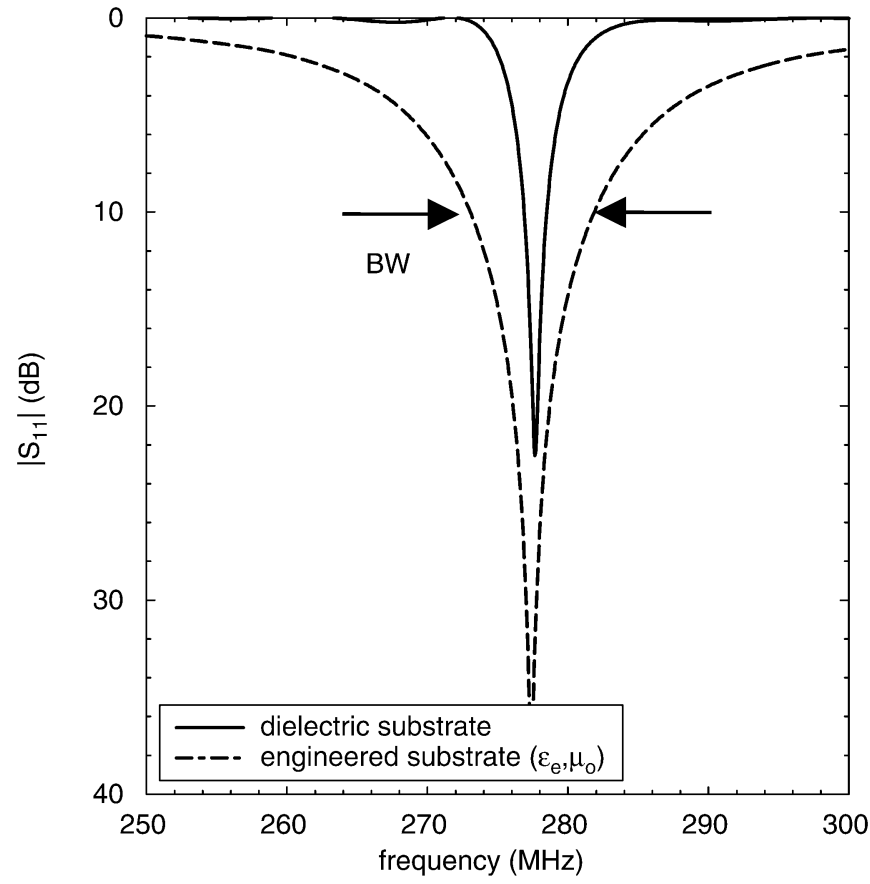

(a)
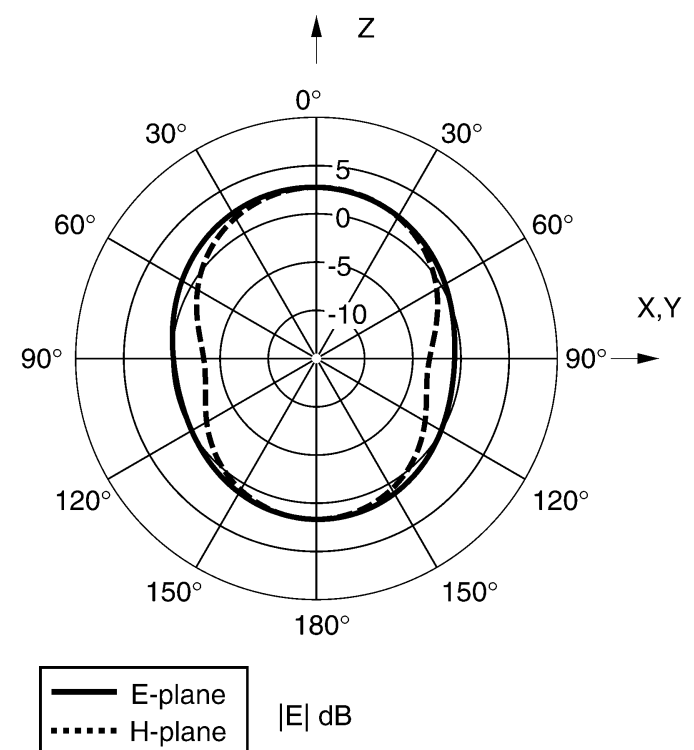

Fig. 8. Patch antenna over the engineered magneto-dielectric meta-substrate: (a) return loss and (b) radiation patterns. The magneto-dielectric substrate enhances the antenna bandwidth significantly (about $600 \%$ ).

of the antenna is $D_{0}=2.9 \mathrm{~dB}$ and it has a front-to-back ratio $1.3 \mathrm{~dB}$ (ground plane size is $0.18 \lambda_{0} \times 0.18 \lambda_{0}$ ). The antenna efficiency is about $e_{r}=67 \%$. If a magneto-dielectric material with lower magnetic loss tangent around 0.01 is used the efficiency is increased to $82 \%$ where the bandwidth is decreased to $B W=2.8 \%$.

Notice that to achieve the same miniaturization factor utilizing only a dielectric material $\left(\mu_{r}=1\right)$ one must use $\varepsilon_{r}=$
23.7. This reduces the bandwidth to about $B W=0.5 \%$ as shown in Fig. 8(a). The efficiency in this case for a dielectric loss tangent of 0.001 is about $e_{r}=64 \%$.

Therefore, utilizing the magneto-dielectric meta-substrate one can offer a miniaturized wideband planar antenna with high efficiency. The antenna bandwidth for the proposed magneto-dielectric substrate is about six times higher than that of the dielectric substrate. 


\section{CONCLUSION}

In this paper, the applications of magneto-dielectric metamaterials for improving the performance and/or achieving miniaturization of RF passive components are presented. Two examples, namely, high performance EBG structures and miniaturized antennas are considered.

It is shown that the design of EBG structures utilizing magneto-dielectrics has the dual benefit of improving band-gap rejection levels, while achieving size reduction. This was demonstrated using a magneto-dielectric woodpile structure and its performance was compared with a dielectric woodpile.

The magneto-dielectric meta-substrates are also shown to provide a great advantage in the design of miniaturized planar antennas with superior radiation and bandwidth characteristics. Utilizing both dielectric and magnetic materials allows for simultaneous ease of antenna impedance matching and miniaturization. A periodic arrangement of dielectric and magneto-dielectric materials is proposed to effectively obtain an engineered substrate having $\left(\mu_{\text {eff }}>\varepsilon_{\text {eff }}\right)$ for designing a miniaturized patch antenna, which demonstrates a relatively high bandwidth and efficiency.

The AEMT was extended to include dielectric and magnetic materials. This model and the FDTD numerical technique with PBC/PML boundary conditions are used in the analysis and design of the structures considered in this paper.

\section{APPENDIX}

Here the formulations for analyzing the layered anisotropic structure of both dielectric and magnetic materials [Fig. 2(b)] with $\hat{x}$ being the unit normal to the interface between the successive layers, as discussed in Section II-A, are presented. In a homogeneous and anisotropic medium with general $\bar{\varepsilon}$ and $\bar{\mu}$ tensors

$$
\begin{aligned}
& \bar{\varepsilon}=\varepsilon_{0}\left[\begin{array}{lll}
\varepsilon_{11} & \varepsilon_{12} & \varepsilon_{13} \\
\varepsilon_{21} & \varepsilon_{22} & \varepsilon_{23} \\
\varepsilon_{31} & \varepsilon_{32} & \varepsilon_{33}
\end{array}\right] \\
& \bar{\mu}=\mu_{0}\left[\begin{array}{lll}
\mu_{11} & \mu_{12} & \mu_{13} \\
\mu_{21} & \mu_{22} & \mu_{23} \\
\mu_{31} & \mu_{32} & \mu_{33}
\end{array}\right]
\end{aligned}
$$

the following differential matrix equation can be written [19], [20]

$$
\frac{d}{d x} \mathbf{V}=\mathbf{H} \cdot \mathbf{V}
$$

where

$$
\begin{aligned}
& \mathbf{V}=\left[\begin{array}{llll}
E_{y} & E_{z} & H_{y} & H_{z}
\end{array}\right]^{T} \\
& \mathbf{H}=\left[\begin{array}{ll}
\mathbf{H}_{11} & \mathbf{H}_{12} \\
\mathbf{H}_{21} & \mathbf{H}_{22}
\end{array}\right]
\end{aligned}
$$

and as shown in (A.5a)-(A.5d) at the bottom of the page, where $\eta_{0}=\sqrt{\mu_{0} / \varepsilon_{0}}$ is the intrinsic free space impedance. Note that, in case the periodicity axes are either along $y$ or $z$ directions the $\bar{\varepsilon}$ and $\bar{\mu}$ tensors are diagonal matrices.

The eigensolutions of (A.2) can be found by letting

$$
\mathbf{V}(x, y, z)=\mathbf{V}_{0} e^{-j k_{x} x} \cdot e^{-j\left(k_{y}^{i} y+k_{z}^{i} z\right)}
$$

The substituting (A.6) in (A.2) yields

$$
\left(\mathbf{H}+j k_{x} \mathbf{I}\right) \cdot \mathbf{V}_{0}=0
$$

where $\mathbf{I}$ is the identity matrix. It is obvious from (A.7) that eigenvalues of $\mathbf{H}$ are the possible propagation constants and its eigenvectors are the propagation modes. The eigenvectors in each layer correspond to both waves propagating in positive and negative $x$ directions. In general the tangential field vector in the $i$ th layer may be written as

$$
\mathbf{V}_{i}(x, y, z)=\mathbf{a}_{i} \cdot e^{-j k_{i x} x} \cdot \mathbf{A}_{i} e^{-j\left(k_{y}^{i} y+k_{z}^{i} z\right)}
$$

where $a_{i}$ is a $4 \times 4$ matrix containing the eigenvectors $\mathbf{a}_{\text {in }}$ 's of matrix $\mathbf{H}$, i.e.

$$
\mathbf{a}_{i}=\left[\begin{array}{llll}
\mathbf{a}_{i 1} & \mathbf{a}_{i 2} & \mathbf{a}_{i 3} & \mathbf{a}_{i 4}
\end{array}\right]
$$

$$
\begin{aligned}
& \mathbf{H}_{11}=j\left[\begin{array}{ll}
k_{y}^{i} \frac{\varepsilon_{12}}{\varepsilon_{11}}+k_{z}^{i} \frac{\mu_{31}}{\mu_{11}} & k_{y}^{i}\left(\frac{\varepsilon_{13}}{\varepsilon_{11}}-\frac{\mu_{31}}{\mu_{11}}\right) \\
k_{z}^{i}\left(\frac{\varepsilon_{12}}{\varepsilon_{11}}-\frac{\mu_{21}}{\mu_{11}}\right) & k_{y}^{i} \frac{\mu_{21}}{\mu_{11}}+k_{z}^{i} \frac{\varepsilon_{13}}{\varepsilon_{11}}
\end{array}\right] \\
& \mathbf{H}_{12}=j\left[\begin{array}{ll}
-\eta_{0} \frac{k_{y}^{i} k_{z}^{i}}{k_{0} \varepsilon_{11}}+\eta_{0} k_{0}\left(\frac{\mu_{31} \mu_{12}}{\mu_{11}}-\mu_{32}\right) & \frac{k_{y}^{i}}{\omega \varepsilon_{11}}+\eta_{0} k_{0}\left(\frac{\mu_{31} \mu_{13}}{\mu_{11}}-\mu_{33}\right) \\
-\eta_{0} \frac{k_{z}^{i 2}}{k_{0} \varepsilon_{11}}+\eta_{0} k_{0}\left(\mu_{22}-\frac{\mu_{21} \mu_{12}}{\mu_{11}}\right) & \eta_{0} \frac{k_{y}^{i} k_{z}^{i}}{k_{0} \varepsilon_{11}}+\eta_{0} k_{0}\left(\mu_{23}-\frac{\mu_{21} \mu_{13}}{\mu_{11}}\right)
\end{array}\right] \\
& \mathbf{H}_{21}=j\left[\begin{array}{ll}
\frac{k_{y}^{i} k_{z}^{i}}{\eta_{0} k_{0} \mu_{11}}+\frac{k_{0}}{\eta_{0}}\left(\varepsilon_{32}-\frac{\varepsilon_{31} \varepsilon_{12}}{\varepsilon_{11}}\right) & -\frac{k_{y}^{i 2}}{\omega \mu_{11}}-\frac{k_{0}}{\eta_{0}}\left(\frac{\varepsilon_{31} \varepsilon_{13}}{\varepsilon_{11}}-\varepsilon_{33}\right) \\
\frac{k_{z}^{i}}{\eta_{0} k_{0} \mu_{11}}+\frac{k_{0}}{\eta_{0}}\left(\frac{\varepsilon_{21} \varepsilon_{12}}{\varepsilon_{11}}-\varepsilon_{22}\right) & -\frac{k_{y}^{i} k_{z}^{i}}{\eta_{0} k_{0} \mu_{11}}-\frac{k_{0}}{\eta_{0}}\left(\varepsilon_{23}-\frac{\varepsilon_{21} \varepsilon_{13}}{\varepsilon_{11}}\right)
\end{array}\right] \\
& \mathbf{H}_{22}=j\left[\begin{array}{cc}
k_{y}^{i} \frac{\mu_{12}}{\mu_{11}}+k_{z}^{i} \frac{\varepsilon_{31}}{\varepsilon_{11}} & -k_{y}^{i}\left(\frac{\varepsilon_{31}}{\varepsilon_{11}}-\frac{\mu_{13}}{\mu_{11}}\right) \\
-k_{z}^{i}\left(\frac{\varepsilon_{21}}{\varepsilon_{11}}-\frac{\mu_{12}}{\mu_{11}}\right) & k_{y}^{i} \frac{\varepsilon_{21}}{\varepsilon_{11}}+k_{z}^{i} \frac{\mu_{13}}{\mu_{11}}
\end{array}\right]
\end{aligned}
$$


$\mathbf{A}_{i}$ is a column vector of coefficients $\mathbf{A}_{\text {in }}$ 's, and

$$
e^{-j \mathbf{k}_{i x} x}=\left[\begin{array}{cccc}
e^{j k_{i x 1} x} & 0 & 0 & 0 \\
0 & e^{j k_{i x 2} x} & 0 & 0 \\
0 & 0 & e^{-j k_{i x 3} x} & 0 \\
0 & 0 & 0 & e^{-j k_{i x 4} x}
\end{array}\right]
$$

Here $k_{i x n}$ 's are determined from the eigenvalues of matrix $\mathbf{H}$. The normal components of the electromagnetic fields are calculated from $\mathbf{V}$ in the following form:

$$
\left[\begin{array}{l}
E_{x} \\
H_{x}
\end{array}\right]=\left[\begin{array}{cccc}
-\frac{\varepsilon_{12}}{\varepsilon_{11}} & -\frac{\varepsilon_{13}}{\varepsilon_{11}} & \eta_{0} \frac{k_{z}^{i}}{k_{0} \varepsilon_{11}} & -\eta_{0} \frac{k_{y}^{i}}{k_{0} \varepsilon_{11}} \\
-\frac{k_{z}^{i}}{\eta_{0} k_{0} \mu_{11}} & \frac{k_{y}^{i}}{\eta_{0} k_{0} \mu_{11}} & -\frac{\mu_{12}}{\mu_{11}} & -\frac{\mu_{13}}{\mu_{11}}
\end{array}\right] \cdot \mathbf{V} .
$$

The electromagnetic fields in terms of the negative and positive propagating waves are given by

$$
\begin{aligned}
\mathbf{V}_{i}(x, y, z)= & {\left[\mathbf{a}_{i,-} \cdot e^{j \mathbf{k}_{i x,-}\left(x-x_{i}\right)} \cdot \mathbf{A}_{i,-}+\mathbf{a}_{i,+}\right.} \\
& \left.\cdot e^{-j \mathbf{k}_{i x,+}\left(x-x_{i}\right)} \cdot \mathbf{A}_{i,+}\right] \cdot e^{-j\left(k_{y}^{i} y+k_{z}^{i} z\right)}
\end{aligned}
$$

where $x_{i}$ is the location of the $i$ th layer, and

$$
\begin{aligned}
\mathbf{a}_{i,-} & =\left[\begin{array}{ll}
\mathbf{a}_{i 1} & \mathbf{a}_{i 2}
\end{array}\right], \quad \mathbf{a}_{i,+}=\left[\begin{array}{ll}
\mathbf{a}_{i 3} & \mathbf{a}_{i 4}
\end{array}\right] \\
\mathbf{A}_{i,-} & =\left[\begin{array}{ll}
A_{i 1} & A_{i 2}
\end{array}\right]^{T}, \quad \mathbf{A}_{i,+}=\left[\begin{array}{ll}
A_{i 3} & A_{i 4}
\end{array}\right]^{T} \\
e^{j \mathbf{k}_{i x,-} x} & =\left[\begin{array}{cc}
e^{j k_{i x 1} x} & 0 \\
0 & e^{j k_{i x 2} x}
\end{array}\right] \\
e^{-j \mathbf{k}_{i x,+} x} & =\left[\begin{array}{cc}
e^{-j k_{i x 3} x} & 0 \\
0 & e^{-j k_{i x 4} x}
\end{array}\right] .
\end{aligned}
$$

In terms of the reflection coefficient matrix $\widehat{\mathbf{R}}_{i, j+1}$ between the $i$ th and $(i+1)$ th regions, $\mathbf{V}_{i}$ can be written as

$$
\begin{aligned}
\mathbf{V}_{i}(x, y, z)=\left[\mathbf{a}_{i,-} \cdot e^{j \mathbf{k}_{i x,-}\left(x-x_{i}\right)}+\mathbf{a}_{i,+}\right. \\
\left.\cdot e^{-j \mathbf{k}_{i x,+}\left(x-x_{i}\right)} \cdot \widehat{\mathbf{R}}_{i, i+1}\right] \cdot A_{i,-} e^{-j\left(k_{y}^{i} y+k_{z}^{i} z\right)} .
\end{aligned}
$$

Applying the continuity of tangential fields one can obtain the reflection coefficient as

$$
\begin{aligned}
\widehat{\mathbf{R}}_{i, i+1}= & \mathbf{R}_{i, i+1}+\mathbf{T}_{i+1, i} \cdot e^{-j \mathbf{k}_{(i+1) x,+} d_{i+1}} \cdot \widehat{\mathbf{R}}_{i+1, i+2} \\
& \cdot\left[\mathbf{I}-e^{-j \mathbf{k}_{(i+1) x,-} d_{i+1}} \cdot \mathbf{R}_{i+1, i} \cdot e^{-j \mathbf{k}_{(i+1) x,+} d_{i+1}}\right. \\
& \left.\cdot \widehat{\mathbf{R}}_{i+1, i+2}\right]^{-1} \\
& \cdot e^{-j \mathbf{k}_{(i+1) x,-} d_{i+1}} \cdot \mathbf{T}_{i, i+1}
\end{aligned}
$$

where $\mathbf{R}_{n, m}$ and $\mathbf{T}_{n, m}$ are the reflection and transmission coefficients at the interface between the $n$th and $m$ th layers assuming each medium is semi-infinite. Also

$$
\begin{aligned}
\mathbf{A}_{i+1,-}= & {\left[\mathbf{I}-e^{-j \mathbf{k}_{(i+1) x,-} d_{i+1}} \cdot \mathbf{R}_{i+1, i}\right.} \\
& \left.\cdot e^{-j \mathbf{k}_{(i+1) x,+} d_{i+1}} \cdot \widehat{\mathbf{R}}_{i+1, i+2}\right]^{-1} \\
& \cdot e^{-j \mathbf{k}_{(i+1) x,-} d_{i+1}} \cdot \mathbf{T}_{i, i+1} \cdot \mathbf{A}_{i,-} \\
= & \mathbf{S}_{i+1, i} \cdot \mathbf{A}_{i,-} .
\end{aligned}
$$

Equations (A.15) and (A.16) are the basic tools to analytically obtain the reflection/transmission coefficients of the layered anisotropic medium.

\section{REFERENCES}

[1] H. Mosallaei and Y. Rahmat-Samii, "Periodic band-gap and effective dielectric materials in electromagnetics: Characterization and applications in nanocavities and waveguides," IEEE Trans. Antennas Propagat., vol. 51, pp. 549-563, Mar. 2003.

[2] X. Gong, W. J. Chappell, and L. P. B. Katehi, "Embedded radiating filters in metamaterial substrates," in Proc. IEEE AP-S Int. Symp. USNC/CNC/URSI National Radio Science Meeting, Columbus, $\mathrm{OH}$, June 22-27, 2003.

[3] T. Le Nadan, J. P. Coupez, S. Toutain, and C. Person, "Optimization and miniaturization of a filter/antenna multi-function module using a composite ceramic-foam substrate," in Proc. IEEE MTT-S Int. Symp., Anaheim, CA, June 12-19, 1999.

[4] J. S. Colburn and Y. Rahmat-Samii, "Patch antennas on externally perforated high dielectric constant substrates," IEEE Trans. Antennas Propagat., vol. 47, pp. 1785-1794, Dec. 1999.

[5] J. D. Joannopoulos, R. D. Meade, and J. N. Winn, Photonic Crystals: Molding the Flow of Light. Princeton, NJ: Princeton Univ. Press, 1995.

[6] Y. Rahmat-Samii and H. Mosallaei, "Electromagnetic band-gap structures: Classification, characterization, and applications," in Proc. 11th Int. Conf. Antennas and Propagation, vol. 2, Manchester, U.K., Apr. 17-20, 2001, pp. 560-564.

[7] R. K. Mongia, A. Ittipiboon, and M. Cuhaci, "Low profile dielectric resonator antennas using a very high permittivity material," Electron. Lett., vol. 30, no. 17, pp. 1362-1363, Aug. 1994

[8] S. Datta, C. T. Chan, K. M. Ho, and C. M. Soukoulis, "Effective dielectric constant of periodic composite structures," Phys. Rev. B., vol. 48, no. 20, pp. 14 936-14 943, Nov. 1993.

[9] T. Søndergaard, J. Broeng, A. Bjarklev, K. Dridi, and S. E. Barkou, "Suppression of spontaneous emission for a two-dimensional honeycomb photonic bandgap structure estimated using a new effective-index model," IEEE J. Quantum Electron., vol. 34, pp. 2308-2313, Dec. 1998.

[10] K. Sarabandi, "Simulation of a periodic dielectric corrugation with an equivalent anisotropic layer," Int. J. Infrared and Millimeter Waves, vol. 11, no. 11, pp. 1303-1321, Nov. 1990

[11] R. E. Collin, "A simple artificial anisotropic dielectric medium," IEEE Trans. Microwave Theory Tech., vol. 6, pp. 206-209, Apr. 1958.

[12] — "A simple artificial anisotropic dielectric medium (Correction)," IEEE Trans. Microwave Theory Tech., vol. 6, pp. 414-414, Oct. 1958.

[13] J. A. Kong, Electromagnetic Wave Theory. New York: Wiley, 1986.

[14] A. Taflove and S. C. Hagness, Computational Electrodynamics: The Finite-Difference Time-Domain Method. Norwood, MA: Artech House, 1995.

[15] A. Taflove, Advances in Computational Electrodynamics: The Finite-Difference Time-Domain Method. Norwood, MA: Artech House, 1998.

[16] H. Mosallaei and Y. Rahmat-Samii, "Broadband characterization of complex periodic EBG structures: An FDTD/prony technique based on the split-field approach," Electromagn. J., vol. 23, no. 2, pp. 135-151, Feb.-Mar. 2003.

[17] K. M. Ho, C. T. Chan, C. M. Soukoulis, R. Biswas, and M. Sigalas, "Photonic band gaps in three dimensions: New layer-by-layer periodic structures," Solid State Commun., vol. 89, no. 5, pp. 413-416, 1994. 
[18] R. C. Hansen and M. Burke, "Antennas with magneto-dielectrics," Microwave Opt. Tech. Lett., vol. 26, no. 2, pp. 75-78, July 2000.

[19] W. C. Chew, Waves and Fields in Inhomogeneous Media, NY: IEEE Press, 1995.

[20] M. A. Morgan, D. L. Fisher, and E. A. Milne, "Electromagnetic scattering by stratified inhomogeneous anisotropic media," IEEE Trans. Antennas Propagat., vol. 35, pp. 191-197, Feb. 1987.

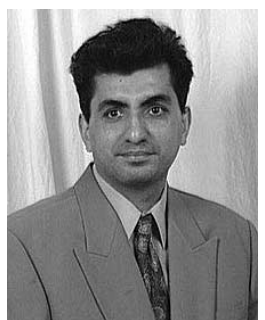

Hossein Mosallaei ( $\left.\mathrm{S}^{\prime} 98\right)$ received the B.Sc. and M.Sc. degrees in electrical engineering from Shiraz University, Shiraz, Iran, and the Ph.D. degree in electrical engineering from University of California, Los Angeles (UCLA), in 1991, 1994, and 2001, respectively.

From 1995 to 1997, he was with the Electrical Engineering Department, Shiraz University. From 1997 to 2001, he was a Research Assistant and Teaching Associate at UCLA. Currently, he is a Research Scientist in the Department of Electrical Engineering and Computer Science, University of Michigan.His research interests include meta-materials, smart RF/wireless systems, microelectromagnetics, EBG and periodic structures, high-frequency circuits, bioengineering, and computational EM.

Dr. Mosallaei is a member of the International Scientific Radio Union (URSI) Commission B. He was the recipient of student prize paper awards in AP-S 2000, 2001, and 2003, a URSI Young Scientist Award in 2001, and an RMTG award in 2002. He was the organizer of radiation laboratory seminars at the University of Michigan in 2002 and 2003. He has also served as Chairman and Co-chairman of several national and international symposia.

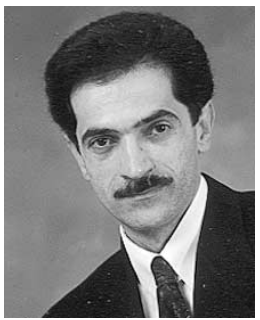

Kamal Sarabandi (S'87-M'90-SM'92-F'00) received the B.S. degree in electrical engineering from Sharif University of Technology, Tehran, Iran, in 1980, the M.S. degree in electrical engineering/mathematics, and the Ph.D. degree in electrical engineering from The University of Michigan, Ann Arbor, in 1986 and 1989, respectively.

$\mathrm{He}$ is Director of the Radiation Laboratory and a Professor in the Department of Electrical Engineering and Computer Science, The University of Michigan. He has 20 years of experience with wave propagation in random media, communication channel modeling, microwave sensors, and radar systems and is leading a large research group consisting of four research scientists, ten Ph.D. students, and two M.S. students. Over the past ten years, he has graduated $15 \mathrm{Ph} . \mathrm{D}$. students. He has served as the Principal Investigator on many projects sponsored by NASA, JPL, ARO, ONR, ARL, NSF, DARPA, and numerous industries. He has published many book chapters and more than 105 papers in refereed journals on electromagnetic scattering, random media modeling, wave propagation, antennas, microwave measurement techniques, radar calibration, inverse scattering problems, and microwave sensors. He has had more than 220 papers and invited presentations in national and international conferences and symposia on similar subjects. His research areas of interest include microwave and millimeter-wave radar remote sensing, electromagnetic wave propagation, and antenna miniaturization.

Dr. Sarabandi is a Member of URSI Commission F and of The Electromagnetic Academy. He received the Henry Russel Award from the Regent of The University of Michigan (the highest honor the University of Michigan bestows on a faculty member at the assistant or associate level). In 1999, he received a GAAC Distinguished Lecturer Award from the German Federal Ministry for Education, Science, and Technology. He also received a 1996 Teaching Excellence Award from the Department of Electrical Engineering and Computer Science, and the 2003/2004 College of Engineering Research Excellence Award, The University of Michigan. He is a Vice President of the IEEE Geoscience and Remote Sensing Society (GRSS), a past Chairman of the Awards Committee of the IEEE GRSS from 1998 to 2002, and a Member of the IEEE Technical Activities Board Awards Committee from 2000 to 2002. He is an Associate Editor of the IEEE TRANSACTIONS ON ANTENNAS AND PROPAGATION and the IEEE SENSORS JOURNAL. He is listed in American Men \& Women of Science, Who's Who in America, and Who's Who in Electromagnetics. 